$\operatorname{IPPP} / 08 / 83$

DCPT $/ 08 / 166$

November 3, 2018

\title{
Diffractive processes at the $\mathrm{LHC}^{1}$
}

\author{
A.D. Martin ${ }^{a}$,V.A. Khoze ${ }^{a, b}$ and M.G. Ryskin ${ }^{a, b}$ \\ ${ }^{a}$ Institute for Particle Physics Phenomenology, University of Durham, Durham, DH1 3LE \\ ${ }^{b}$ Petersburg Nuclear Physics Institute, Gatchina, St. Petersburg, 188300, Russia
}

\begin{abstract}
We present a model of high energy soft $p p$ interactions that has multi $s$ - and $t$-channel components, which has been tuned to describe all the available data. The $t$-channel components allow matching of the soft to the hard (QCD) Pomeron. Absorptive effects are found to be large, and, for example, suppress the prediction of the total $p p$ cross section to about $90 \mathrm{mb}$ at the LHC. We use the model to calculate the survival probability, $S^{2}$, of the rapidity gaps in the exclusive process $p p \rightarrow p+H+p$, a process with great advantages for searching for the $H \rightarrow b \bar{b}$ signal. We consider both eikonal and enhanced rescattering.
\end{abstract}

We discuss two general topics. One is an attempt to obtain a self-consistent description of the high energy behaviour of all soft observables, such as $\sigma_{\mathrm{tot}}, d \sigma_{\mathrm{el}} / d t, d \sigma_{\mathrm{SD}} / d t d M^{2}$, particle multiplicities etc., in terms of the underlying physics; and to predict these observables at the LHC. The second topic is to make reliable estimates for the rates of exclusive processes, such as $p p \rightarrow p+A+p$, at the LHC, where $A$ is a heavy object and the + signs denote rapidity gaps. Particularly topical is when $A$ is a Higgs boson which decays into $b \bar{b}$; the mass of $A$ can be measured in the exclusive process with very good accuracy $\left(\Delta M_{A} \sim 1-2 \mathrm{GeV}\right)$ by the missing-mass method by detecting the outgoing forward protons. Moreover, a specific $J_{z}=0$ selection rule [1] significantly reduces the $b \bar{b}$ background and also greatly simplifies the spinparity analysis of $A$. The two topics are inter-related since soft rescattering can destroy the rapidity gaps and strongly deplete the signal. Thus we need a reliable model of soft interactions to estimate the small survival factor $S^{2}$ of the rapidity gaps.

\footnotetext{
${ }^{1}$ Based on a talk by A.D. Martin at Diffraction 2008, La Londe-les-Maures, France, September 2008.
} 


\section{Soft scattering including absorptive effects}

The total and elastic proton-proton cross sections are usually described in terms of an eikonal model, which automatically satisfies $s$-channel elastic unitarity. To account for the possibility of excitation of the initial proton, that is for two-particle intermediate states with the proton replaced by $N^{*}$, we use the Good-Walker formalism [2]. Already at Tevatron energies the absorptive correction to the elastic amplitude, due to elastic eikonal rescattering, gives about a $20 \%$ reduction of simple one Pomeron exchange. After accounting for low-mass proton excitations (that is $N^{*}$ 's in the intermediate states) the correction becomes twice larger (that is, up to $40 \%)$. Next, in order to describe high-mass diffractive dissociation, $d \sigma_{\mathrm{SD}} / d M^{2}$, we have to include an extra factor of 2 from the AGK cutting rules [3]. Thus, the absorptive effects in the triple-Regge domain are expected to be quite large. The previous triple-Regge analyses (see, for example, [4]) did not allow for absorptive corrections and the resulting triple-Regge couplings must be regarded, not as bare vertices, but as effective couplings embodying the absorptive effects. Thus, we perform a new triple-Regge analysis of the fixed-target FNAL, CERN-ISR and Tevatron data that includes the absorptive effects explicitly [5]. The bare triple-Pomeron coupling is found to be about three times larger than before; now $g_{3 P}=\lambda g_{n}$ with $\lambda \simeq 0.2$ where $g_{N}$ is the Pomeron-proton coupling.

\subsection{Inclusion of multi-Pomeron vertices}

Since the triple-Pomeron vertex turns out to be rather large, the contribution of the so-called 'enhanced' diagrams, with a few vertices, is not negligible. Moreover, it is more reasonable to include multi-Pomeron contributions with $n$ to $m$ Pomeron vertices given by $g_{m}^{n} \propto \lambda^{n+m}$ than to assume that $g_{m}^{n}=0$ for $n+m>3$. These effects are included in the following evolution equation for the opacity $\Omega$ in rapidity space, at given impact parameter $b$

$$
\begin{aligned}
\frac{d \Omega_{k}(y, b)}{d y}= & e^{-\lambda \Omega_{i}\left(y^{\prime}, b\right) / 2} e^{-\lambda \Omega_{k}(y, b) / 2}\left(\Delta+\alpha^{\prime} \frac{d^{2}}{d^{2} b}\right) \Omega_{k}(y, b) \\
& <-(\mathrm{c})-><-(\mathrm{b})->\quad<---(\mathrm{a})--->
\end{aligned}
$$

where $y^{\prime}=\ln s-y$. Let us explain the meanings of the three terms on the right hand side of (1). If only the last factor, (a), is present then the evolution generates the ladder-type structure of the bare Pomeron exchange amplitude, where the Pomeron trajectory $\alpha_{P}=1+\Delta+\alpha^{\prime} t$. The inclusion of (b) allows for rescatterings of an intermediate parton $c$ with the "target" proton $k$; Fig. 1(a) shows the simplest (single) rescattering which generates the triple-Pomeron diagram. Finally, (c) allows for rescatterings with the beam $i$. In this way we include the absorptive effects generated by all multi-Pomeron diagrams like the one shown in Fig. 1(b). There is an analogous equation for the evolution of $\Omega_{i}(y, b)$, and the two equations may be solved iteratively. A detailed discussion can be found in [6], where $\alpha^{\prime}$ is set to zero. 
(a)

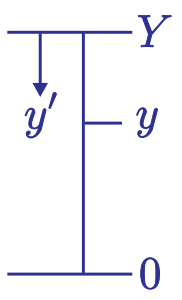

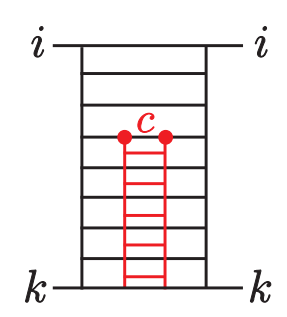

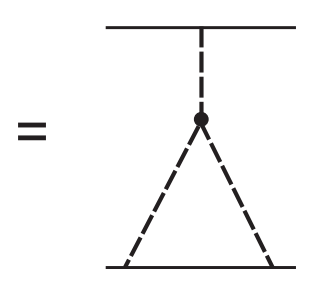

(b)

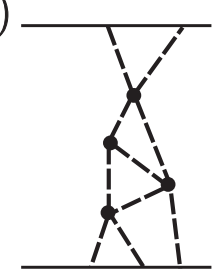

Figure 1: (a) The ladder structure of the triple-Pomeron amplitude between diffractive eigenstates $i, k$ of the proton; the rapidity $y$ spans an interval 0 to $\mathrm{Y}=\ln$. (b) A multi-Pomeron diagram.

\subsection{Inclusion of different $t$-channel components}

Besides allowing for $\alpha^{\prime} \neq 0$, a new development is an analysis [7] in which we allow for four different $t$-channel states, which we label $a$ : one for the secondary Reggeon $(R)$ trajectory and three Pomeron states $\left(P_{1}, P_{2}, P_{3}\right)$ to mimic the BFKL diffusion in the logarithm of parton transverse momentum, $\ln \left(k_{t}\right)$ [8]. To be precise, since the BFKL Pomeron [9] is not a pole in the complex $j$-plane, but a branch cut, we approximate the cut by three $t$-channel states of a different size. The typical values of $k_{t}$ in each of the three states is about $k_{t 1} \sim 0.5 \mathrm{GeV}$, $k_{t 2} \sim 1.5 \mathrm{GeV}$ and $k_{t 3} \sim 5 \mathrm{GeV}$. Thus (1) is replaced by

$$
\frac{d \Omega_{k}^{a}(y, b)}{d y}=e^{-\lambda \Omega_{i}\left(y^{\prime}, b\right) / 2} e^{-\lambda \Omega_{k}(y, b) / 2}\left(\Delta^{a}+\alpha^{\prime} \frac{d^{2}}{d^{2} b}\right) \Omega_{k}^{a}(y, b)+V_{a a^{\prime}} \Omega_{k}^{a^{\prime}} .
$$

where $\Delta^{a}=\alpha_{a}(0)-1$ and $\alpha_{a}^{\prime}=\alpha_{P}^{\prime}$ for $a=P_{1}, P_{2}, P_{3}$, while for the secondary Reggeon, $(a=R)$, which is built of quarks, we take $\Delta^{R}=\alpha_{R}(0)=0.6$ and $\alpha_{R}^{\prime}=0.9 \mathrm{GeV}^{-2}$. The transition factors $V_{a a^{\prime}}$ were fixed by properties of the BFKL equation. In the exponents, the opacities $\Omega_{i}\left(\Omega_{k}\right)$ are actually the sum of the opacities $\Omega_{i}^{a^{\prime}}\left(\Omega_{k}^{a^{\prime}}\right)$ with corresponding coefficients.

Such a model allows us to reproduce all the available data on diffractive cross sections, $\sigma_{\text {tot }}, d \sigma_{\mathrm{el}} / d t, \sigma_{\mathrm{SD}}^{\text {low mass }}, d \sigma_{\mathrm{SD}} / d M^{2}$. We find that the triple-Pomeron coupling parameter $\lambda=$ 0.25 and that $\Delta^{a}=0.3$ for all three components of the Pomeron, consistent with the expectations of resummed NLL BFKL which gives $\omega_{0} \equiv \alpha_{P}(0)-1 \sim 0.3$ pratically independent of $k_{t}[10]$. The slopes, $\alpha_{P}^{\prime}$, are $0.05,0.005$ and $0 \mathrm{GeV}^{-2}$ for the large, intermediate and small size components of the Pomeron respectively.

The model allows us, in principle, to predict all features of soft $p p$ high energy interactions. Examples are shown in Fig. 1.2. Note that, due to absorptive effects, the total cross section at the LHC energy $\sqrt{s}=14 \mathrm{TeV}$ is predicted to be only about $90 \mathrm{mb}$. Also, the multiplicities of the secondaries produced by the $t$-channel Pomeron components of different sizes, are shown in Fig. 1.2(d). We see that, starting with the same 'bare' intercepts $(\Delta=0.3)$, after the absorptive correction, the contribution of the large-size component becomes practically flat, while the small-size contribution, which is much less affected by the absorption, continues to 

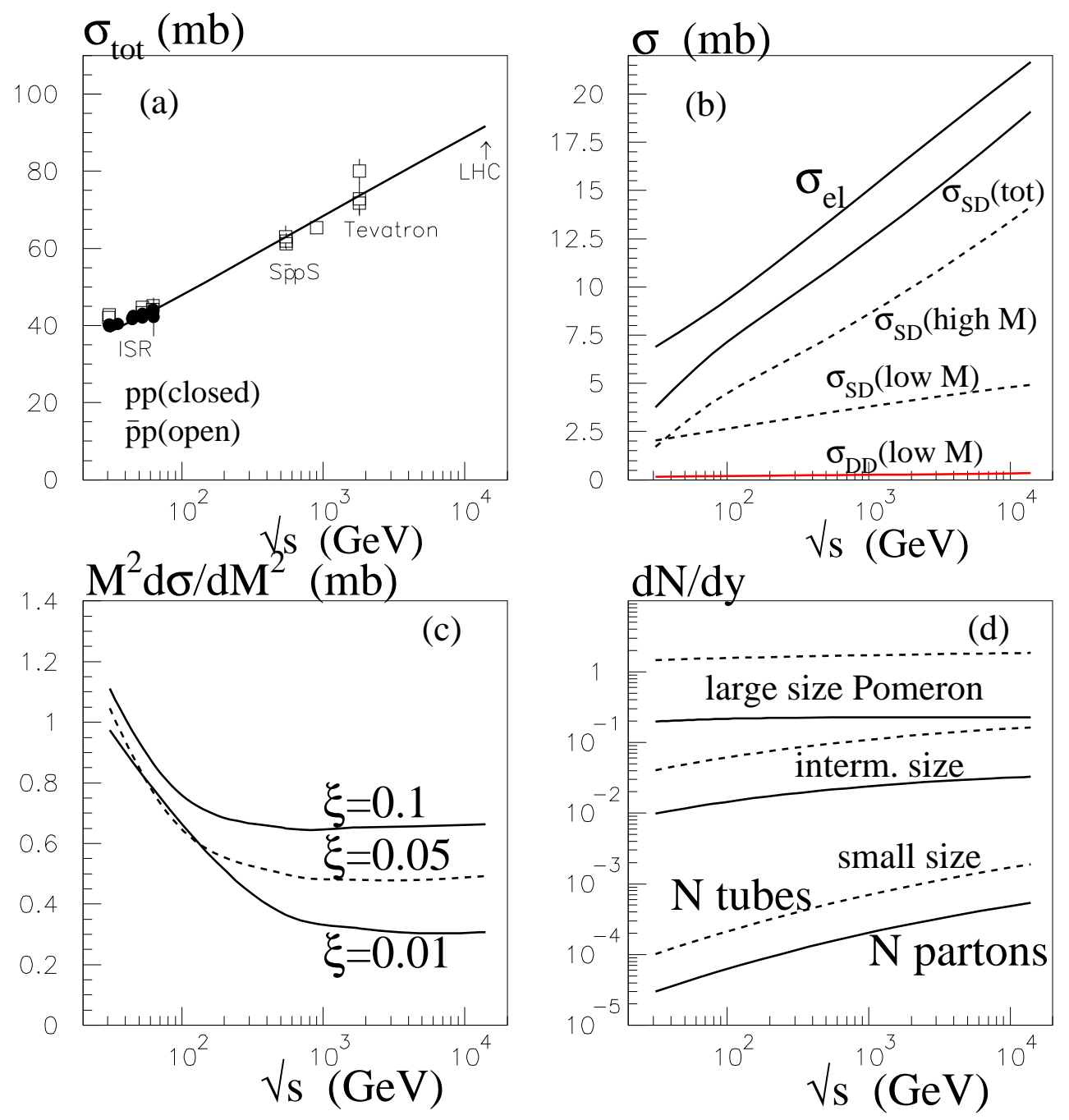

Figure 2: The energy dependence of (a) the total, (b) the elastic and diffractive dissociation, $p p$ cross sections and (c) the cross sections of dissociation to a fixed $M^{2}$ state, where $\xi=M^{2} / s$. Plot (d) shows the parton multiplicity (solid lines) and the number of 'colour tubes' (dashed) produced by the Pomeron components of different size. 


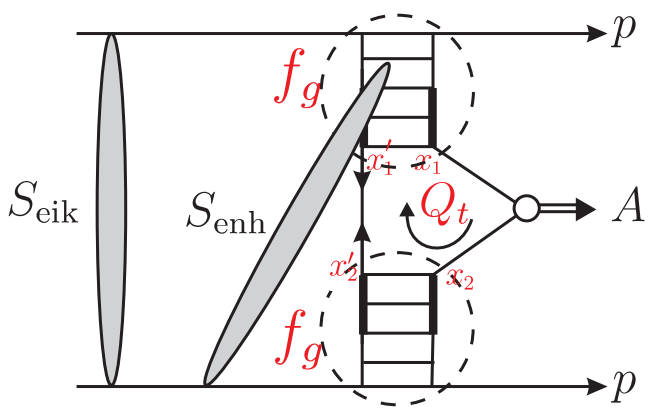

Figure 3: The mechanism for the exclusive process $p p \rightarrow p+A+p$, with the eikonal and enhanced survival factors shown symbolically. The thick lines on the Pomeron ladders, either side of the subprocess $(g g \rightarrow A)$, indicate the rapidity interval $\Delta y$ where enhanced absorption is not permitted [14].

grow with energy. Such a behaviour is consistent with experiment, where the density of low $k_{t}$ secondaries is practically saturated, while the probability to produce a hadron with a large transverse momentum (say, more than $5 \mathrm{GeV}$ ) grows with the initial energy.

\section{Exclusive Processes}

We have already emphasised the value of the observation of an exclusive process of the type $p p \rightarrow p+A+p$ at the LHC $[11,12,13]$. The process is sketched in Fig. 3. The case of $A=H \rightarrow b \bar{b}$ is particularly interesting. The cross section is usually written in the form

$$
\sigma(p p \rightarrow p+A+p) \sim \frac{\left\langle S^{2}\right\rangle}{B^{2}}\left|N \int \frac{d Q_{t}^{2}}{Q_{t}^{4}} f_{g}\left(x_{1}, x_{1}^{\prime}, Q_{t}^{2}, \mu^{2}\right) f_{g}\left(x_{2}, x_{2}^{\prime}, Q_{t}^{2}, \mu^{2}\right)\right|^{2}
$$

where $B / 2$ is the $t$-slope of the proton-Pomeron vertex, and the constant $N$ is known in terms of the $A \rightarrow g g$ decay width. The amplitude-squared factor, $|\ldots|^{2}$, can be calculated in perturbative QCD, since the dominant contribution to the integral comes from the region $\Lambda_{Q C D}^{2} \ll Q_{t}^{2} \ll M_{A}^{2}$, for the large values of $M_{A}^{2}$ of interest. The probability amplitudes, $f_{g}$, to find the appropriate pairs of $t$-channel gluons $\left(x_{1}, x_{1}^{\prime}\right)$ and $\left(x_{2}, x_{2}^{\prime}\right)$ of Fig. 3 , are given by skewed unintegrated gluon densities at a hard scale $\mu \sim M_{A} / 2$. To evaluate the cross section of such an exclusive processes it is important to know the probability, $\left\langle S^{2}\right\rangle$, that the rapidity gaps survive and will not be filled by secondaries from eikonal and enhanced rescattering effects.

The model of soft interactions described above gives a gap survival probability $\left\langle S_{\text {eik }}^{2}\right\rangle \sim$ $0.012^{2}$ with respect to the eikonal (including the elastic and low-mass proton excitation) rescattering, for the exclusive production of a Higgs boson. The model may also be used to calculate

\footnotetext{
${ }^{2}$ If we were to adjust $\left\langle S_{\text {eik }}^{2}\right\rangle$, obtained with the $t$ dependence of our model, to its value corresponding to an exponential slope $B=4 \mathrm{GeV}^{-2}$, then we obtain $\left\langle S_{\text {eik }}^{2}\right\rangle_{\text {eff }}=0.024$, which is consistent with our previous estimate [15], see the discussion below.
} 
the absorptive correction to exclusive cross sections caused by the so-called enhanced diagrams, that is by the interaction with the intermediate partons, see Fig. 3. This rescattering violates 'soft-hard' factorisation, since the probability of such an interaction depends both on the transverse momentum and on the impact parameter of the intermediate parton. The model predicts $\left\langle S_{\text {enh }}^{2}\right\rangle \sim \frac{1}{3}$.

We emphasize that comparing the values of the survival factors in this way is too simplistic. The problem is that, with enhanced screening on intermediate partons, we no longer have exact factorisation between the hard and soft parts of the process. Thus, before computing the effect of soft absorption we must fix what is included in the bare exclusive amplitude calculated in terms of perturbative QCD. Two observations are importrant.

The first observation is that the bare amplitude is calculated as a convolution of two generalised (skewed) gluon distributions with the hard subprocess matrix element, see (3). These gluon distributions are determined from integrated gluon distributions of a global parton analysis of mainly deep inelastic scattering data. Now, the phenomenological integrated parton distributions already include the interactions of the intermediate partons with the parent proton. Thus calculations of $S_{\text {enh }}$ should keep only contributions which embrace the hard matrix element of the type shown in Fig. 3.

The second observation is that the phenomenologically determined generalised gluon distributions, $f_{g}$, are usually taken at $p_{t}=0$ and then the observed "total" exclusive cross section is calculated by integrating over $p_{t}$ of the recoil protons assuming the an exponential behaviour $e^{-B p_{t}^{2}}$; that is

$$
\int d p_{t}^{2} e^{-B p_{t}^{2}}=1 / B=\left\langle p_{t}^{2}\right\rangle .
$$

However, the total soft absorptive effect changes the $p_{t}$ distribution in comparison to that for the bare cross section determined from perturbative QCD. Thus the additional factor introduced by the soft interactions is not just the gap survival $S^{2}$, but rather the factor $S^{2} / B^{2}[11,16]$, which strictly speaking has the form $S^{2}\left\langle p_{t}^{2}\right\rangle^{2}$.

In order to compare determinations of the suppression due to absorptive effects we should compare only the values of the complete cross section for $p p \rightarrow p+A+p$. However, a comparison is usually made by reducing the cross section to a factorized form. If this is done, as in (3), then the predictions for the survival factor to eikonal and enhanced screening of the exclusive production of a $120 \mathrm{GeV}$ Higgs at the LHC are $\left\langle S^{2}\right\rangle_{\mathrm{eff}}=0.004,0.009,0.015$ where enhanced screening is only permitted outside a threshold rapidity gap [14] $\Delta y=0,1.5,2.3$ respectively, see Fig. 3. The values correspond to $B=4 \mathrm{GeV}^{-2}$. That is, allowing for the threshold effect, we predict $\left\langle S^{2}\right\rangle_{\text {eff }} \simeq 0.015 \pm 0.01$.

\section{Summary}

We have described a model, tuned to the existing data, which is capable of predicting all features of high energy soft $p p$ interactions. Absorptive effects are found to be large. For example, the 
total $p p$ cross section is predicted to be only about $90 \mathrm{mb}$ at the LHC energy $\sqrt{s}=14 \mathrm{TeV}$. We used this model to estimate the exclusive cross section for Higgs production, $p p \rightarrow p+H+p$, at the LHC. We calculated the survival factor of the rapidity gaps to both eikonal and enhanced rescattering and found $\left\langle S^{2}\right\rangle_{\text {eff }} \simeq 0.015 \pm 0.01$. Note that, from exclusive CDF data and leading neutron data at HERA, there is evidence that $\left\langle S_{\text {enh }}^{2}\right\rangle$ is somewhat larger than the estimates obtained here, such that $\left\langle S^{2}\right\rangle_{\text {eff }}$ is nearer the upper limit of the quoted interval. Early LHC runs can measure $\left\langle S_{\text {enh }}^{2}\right\rangle[17]$.

\section{References}

[1] V.A. Khoze, A.D. Martin and M. Ryskin, Eur. Phys. J. C 19 (2001) 477 [Err-ibid. C 20 (2001) 599].

[2] M.L. Good and W.D. Walker, Phys. Rev. 120 (1960) 1857.

[3] V.A. Abramovsky, V.N. Gribov and O.V. Kancheli, Sov. J. Nucl. Phys. 18308 (1973).

[4] R.D. Field and G.C. Fox, Nucl. Phys. B80 367 (1974);

A.B. Kaidalov, V.A.Khoze, Yu.F. Pirogov and N.L. Ter-Isaakyan, Phys. Lett. B45 471 (1974);

for a review see A.B. Kaidalov, Phys. rep. 50157 (1979).

[5] E.G.S. Luna, V.A. Khoze, A.D. Martin and M.G. Ryskin, arXiv:0807.4115.

[6] M.G. Ryskin, A.D. Martin and V.A. Khoze, Eur. Phys. J. C54 199 (2008).

[7] M.G. Ryskin, A.D. Martin and V.A. Khoze, to be published.

[8] L.N. Lipatov, Sov. Phys. JETP 63904 (1986).

[9] V.S. Fadin, E.A. Kuraev, and L.N. Lipatov, Phys. Lett. B 60 (1975) 50;

E.A. Kuraev, L.N. Lipatov, and V.S. Fadin, Zh. Eksp. Teor. Fiz. 71 (1976) 840 [Sov. Phys. JETP 44443 (1976)]; ibid. 72 (1977) 377 [45 (1977) 199];

I.I. Balitsky and L.N. Lipatov, Yad. Fiz. 28 (1978) 1597 [Sov. J. Nucl. Phys. 28 (1978) 822].

[10] V.S. Fadin and L.N. Lipatov, Phys. Lett. B429 (1998) 127;

G. Camici and M. Ciafaloni, Phys. Lett. B430 (1998) 349;

G.P. Salam, JHEP 9807019 (1998); Act. Phys. Plol. B30 3679 (1999);

M. Ciafaloni, D. Colferai and G.P. Salam, Phys. Rev. D60 114036 (1999).

[11] V. A. Khoze, A. D. Martin and M. G. Ryskin, Eur. Phys. J. C 23, 311 (2002).

[12] A. De Roeck, V. A. Khoze, A. D. Martin, R. Orava and M. G. Ryskin, Eur. Phys. J. C 25, 391 (2002). 
[13] M. G. Albrow et al. [FP420 R and D Collaboration], arXiv:0806.0302 [hep-ex].

[14] V.A. Khoze, A.D. Martin and M.G. Ryskin, JHEP 0605036 (2006):

A.B. Kaidalov et al., Eur. Phys. J. C21, 521 (2001).

[15] V.A. Khoze, A.D. Martin and M.G. Ryskin, Eur. Phys. J. C18 (2000) 167.

[16] V. A. Khoze, A. D. Martin, M. G. Ryskin and W. J. Stirling, Eur. Phys. J. C 35, 211 (2004).

[17] V.A. Khoze, A.D. Martin and M.G. Ryskin, Eur. Phys. J. C55 (2008) 363. 